$$
\epsilon_{i}=\beta^{2} \lambda n_{v} z=k \theta^{\prime} z,
$$

\title{
研究工作簡报
}

\section{Stoner 集体电子铁磁理論的圖示}

Stoner 的“集体电子鉄磁性” (Collective Electron Ferromagnetism) 理論最初出現于 1938 -39 年 [1,2], 其中大量数值計算当时已經完成。以厉, Wohlfarth 对此理論加以延伸[3]与应用，判考虑解提 假設的量子力学与粹計力学基础。Stoner 理淪自 d电子能带出認为自磁化强度是由于 $\mathrm{d}$ 一带中林成 对的电子自旋，亦即 $\mathrm{d}$ - 带中未成对的“空穴” (hole) 自旋。此外, Stoner 理論有三个前提假設: (1) d带是一种标准形式, 即带中电子态的悠度正比于能量 的平一方根（对于带中位于較上部的态而言，能量自带 的上限往下量, 即带是对称的); (2) 交换作用能正比 于自發磁化强度的平方, 这相当于 Weiss 分子場; (3)带中粒子服从 Fermi-Dirac 統計。Stoner 理論 提出許各有明显优点的結果，虽然其前提的适实性問 題有所争論 [4]。这里报导的研究工作中, Stoner 理 論的前提暂未討論，而以其为基础提供了一系列能带 圆示。这些圖示鮮明地描繪了 Stoner 理諭的物理情 况。Stoner 絓过繁复計算所得的一个重要結果一 关于磁生铁磁性及磁化是否完全的判据，可利用圆示 简捷地得到。此外，可利用圖示考虑在鉄磁状态下由 d-带分裂引致的自 $d$ 一带至 s- 带的电子迁移，井对在 $0^{\circ} \mathrm{K}$ 的䤼作了估算。关于金屬中同 时存在的 $\mathrm{d}-$ 带 及 s- 带之開的电子迁移, Stoner 完全汥有考虑, Wohlfarth 曾考虑了䤼中的电子迁移[5]，但他的結 果是在没有鉄磁性的前提下得到的, 只能适用于高温 (居里点以上)。

此处仅滆略一述如何利用圖示得到鉄磁制据。圖 中 $\epsilon_{0}$ 是当舞鉄磁性时空穴的最高能量，自 $\mathrm{d}-$ 节上限 $E_{0}$ 往下量。 $\epsilon_{i}$ 为由交作用而来的每一空分的能量,

A

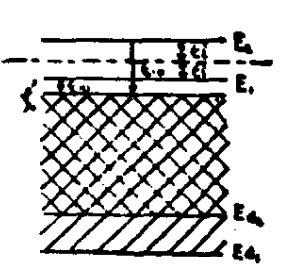

B

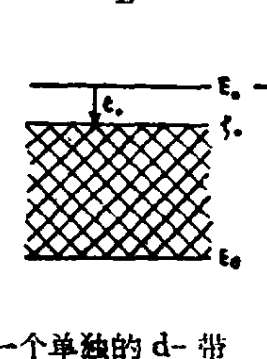

$0^{\circ} \mathrm{K}$ 时一个单独的 $\mathrm{d}-\mathrm{An}$

B: 無鋘磷性

A: $\epsilon_{i}<\epsilon_{0}$ ，而且磁化不完全

C: $\epsilon_{i}>\epsilon_{0}$, 磁化完全
式中 $\beta$ 是 Bohr 磁子， $k$ 是 Boltzmann 桴数， $n_{v}$ 为 物体中单位体积內的空穴数， $z$ 为相对磁化强度，的 $z=I \mid n_{v} E ， I$ 为实际出現的能和自媵位化强度。7. 及 $\theta^{\prime}$ 为每一鉄磁物質的特征参数 (作为常数)，合 $n_{\text {I }}$ 及 $n_{1}$ 分别为自旋正行及反不行于 $I$ 的突穴的数且，则在 圖 A 部分

$$
\begin{aligned}
& n_{2}=\frac{1}{2} \frac{N_{h}}{\epsilon_{0}^{3 / 2}} \epsilon_{30}^{3 / 2}=\frac{1}{2} \frac{N_{h}}{\epsilon^{3 / 2}}\left(\epsilon_{10}+2 \epsilon_{i}\right)^{3 / 2}, \\
& n_{1}=\frac{1}{2} \frac{N_{h}}{\epsilon_{0}^{3 / 2}} \epsilon_{10}^{3 / 2} 。
\end{aligned}
$$

式中 $N_{h}$ 为空究总数。圖及式(1) 和式(䏠) it $\mathrm{S}^{+}$oner 理論的三个前提得到。命 $z_{0}$ 为 $0^{\circ} \mathrm{K}$ 时 $z$ 的傎, 命 $5=$ $k \theta^{\prime} / \epsilon_{0}$, 可知 $\epsilon_{i}=\xi \epsilon_{0} z_{0}$ 。利湖条作 $N_{h}=n_{2}+n_{1}$ 及 $z_{0}=\left(n_{2}-n_{1}\right) / N_{h}$, 守 (2) 式的得

$$
\left[2 \xi z_{0}+\left(1-z_{0}\right)^{2 / 3}\right]^{3 / 2}+\left(1-z_{0}\right)=2 \text { 。 }
$$

当 $\xi$ 值过大以致情况由馗中的 $\mathrm{A}$ 过海圳 $\mathrm{C}$, 式 (3) 不 再成立。当 $z_{0}<1$ 时，式(3)最后可变成

$$
z_{0}^{2}=\frac{27}{40}(3 \xi-2)-O\left(z_{0}^{4}\right),
$$

其中最后一项的意义是数量級为 $z_{0}{ }^{4}$ 。用式(4) 的知: 当 $z_{0} \rightarrow 0$ 时, $3 \xi-2 \rightarrow 0$; 当 $3 \xi-2<0$ 时, $z_{0}$ 不矿能为 实数; 当 $35-2>0$ 时, $z_{0}$ 挎大于等的数值，因此，得:

鉄磁性發生的条件: $3 \xi-2>0$, 即 $\xi>2$ 又自式(3) 叮知, 当 $z_{0}$ 自小于 1 的值趋于 1 时, 左边 趋于 $(2 \xi)^{3 / 2}$, 亦即当 $2 \xi \rightarrow 2^{2 / 3}$ 或 $\xi \rightarrow 2^{-1 / 3}$ 时, $z_{0} \rightarrow 1$ 。 因此, 得:

$$
\text { 当 } \frac{2}{3}<\xi<2^{-1 / 3} \text { 时, } \quad 0<z_{0}<1 \text { 。 }
$$

当 $\xi \geqq 2^{-1 / 3}$ 时，情况由 $\mathrm{A}$ 过海到 $\mathrm{C}, z_{0}$ 总是 1 。式(5) 及 (6) 即 Stoner 的关于碐生鉄磁性及磁化是否光全 的判据。

向仁生

(中国科学院应用物理研究际) 1957年7月2日

[1] E. C. Stoner, Proc. Roy. Soc., A165 (1938), 372.

[2] E. C. Stoner, Proc. Roy. Soc., A169(1939), 339.

[ 3 ] E. P. Wohliarth, Proc. Roy. Soc., A195 (1949), 434.

[4] J. H. Van Vle:k, Proc. IRE, 44(1956), 1248.

[5] E. P. Wohlfarth, Proc. Phys. Soc., 60(1948), 360.

\section{萧甲腈的 Stephen 反应 中間物的晶体結构}

Stephen 反应是由腈洲备相应的莤的一利方

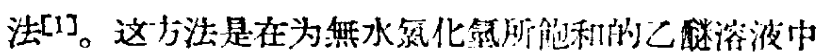
特腈化物利用無水氮化亚錫还原，优戊为一川间化合 
$\mathrm{H}$

物 $\left(\mathrm{R}-\mathrm{C}=\mathrm{NH} \cdot \mathrm{HCl}_{2} \mathrm{SnCl}_{4}\right.$ (Stanric aldimonium chlorides), 这中間物水解后即得醛。对于这 反应的机构曾有不少討論[2,3]。在本工作中，选取了 荣甲腈为对象，抹按一般文献記裁的方法[3]制得化学 式为 $\left(\mathrm{C}_{6} ; \mathrm{H}_{5} \mathrm{CH}=\mathrm{NH} \cdot \mathrm{HCl}\right)_{2}, \mathrm{SnCl}_{4}$ 的单晶体，其会 父的实驗值和部算值各为 $5.41 \%$ 和 $5.16 \%$ 。品体的 Laue 对称性为 $\mathrm{C}_{\mathrm{i}}$ ，按三个方向摄取了晶体的等倾斜 Weissenberg 圖，从而引得三斜晶胞的参数：

$$
\begin{aligned}
& a=7.41 \pm 0.03 \AA \\
& b=7.84 \pm 0.03 \AA \\
& c=10.45 \pm 0.05 \AA \\
& \alpha=86^{\circ} 54^{\prime} \pm 1^{\circ} \\
& \beta=85^{\circ} \pm 1^{\circ} \\
& \gamma=57^{\circ} 54^{\prime} \pm 1^{\circ}
\end{aligned}
$$

晶胞中含一个化学式量, 嘧度的計算值和实驗值备为 1.76 和 $1.737 \pm 0.02$ 克/厘米 3 。

由 $h 0 l$ 和 $0 \mathrm{kl}$ 的强度数据, 計算了 Patterson 投影 $P(u w)$ 和 $P(v w)$ ，由这两張投影推得了重原子 $\mathrm{Sn}$ 和 $\mathrm{Cl}$ 的参数, 抹初步确定昆体的空間群为 $C_{i}^{1}$ 。根 据这些参数抹接 $C_{i}^{1}$ 的对称性計算了电子密度分布因 数 $\rho(x, y), \rho(x, z), \rho(y, z)$, 从而找到了碳原子和筑

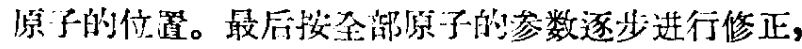
楾到”圖 1 和罚 2 的絬果, 現将备原子的参数列于表 1 中。鼠体的結恎示㤕于圖 3 中。
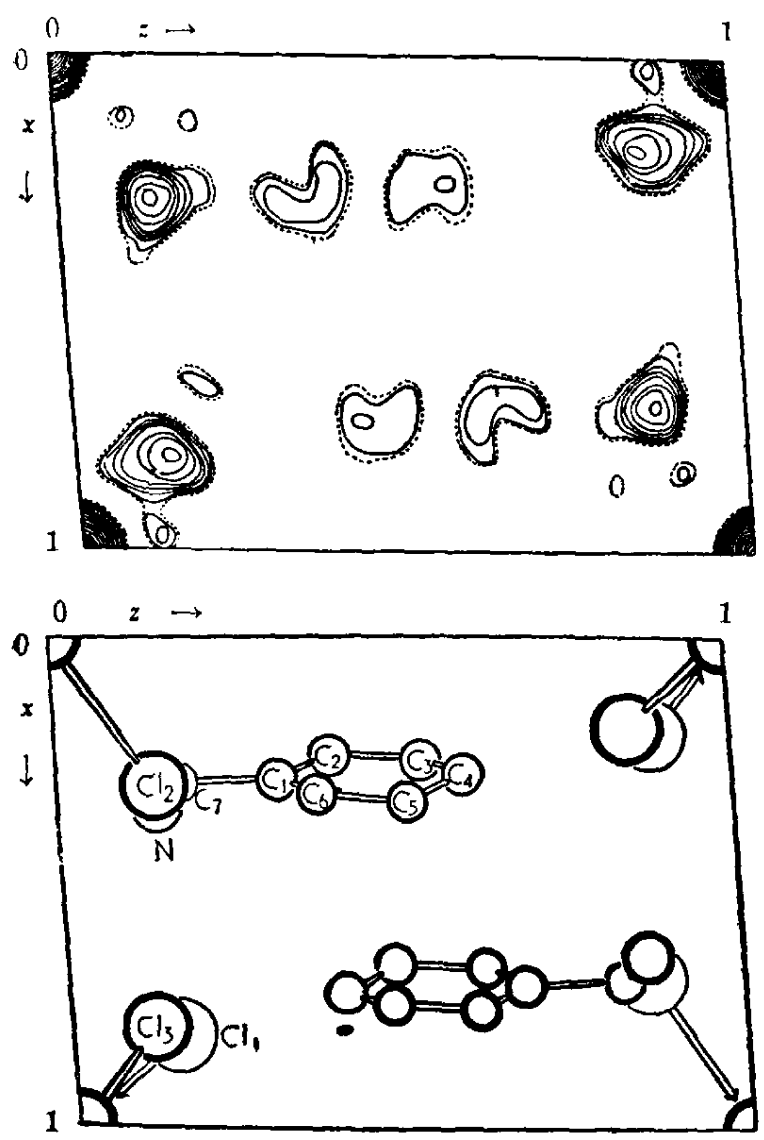

1 电子密度分布函数 $\rho(x, 2)$ 和結构的相应的投影
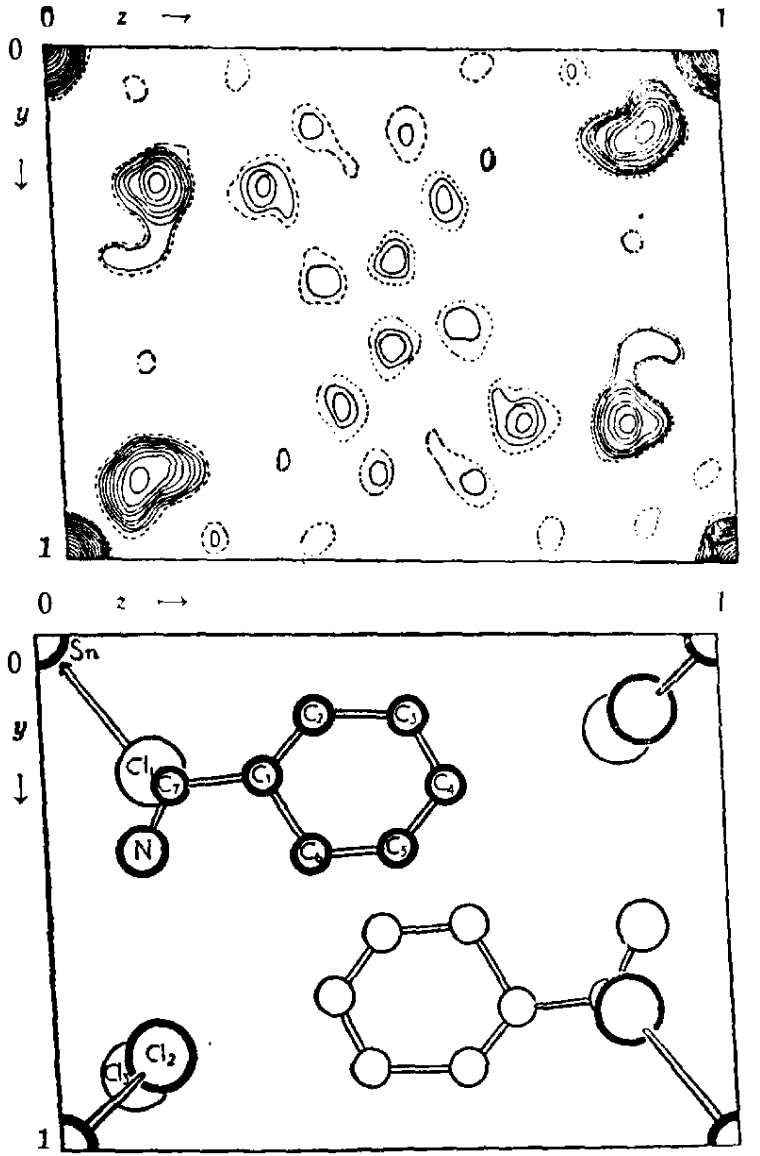

圆 2 毛于密度分有涵数 $\rho(y, z)$ 和結构的相应的投影

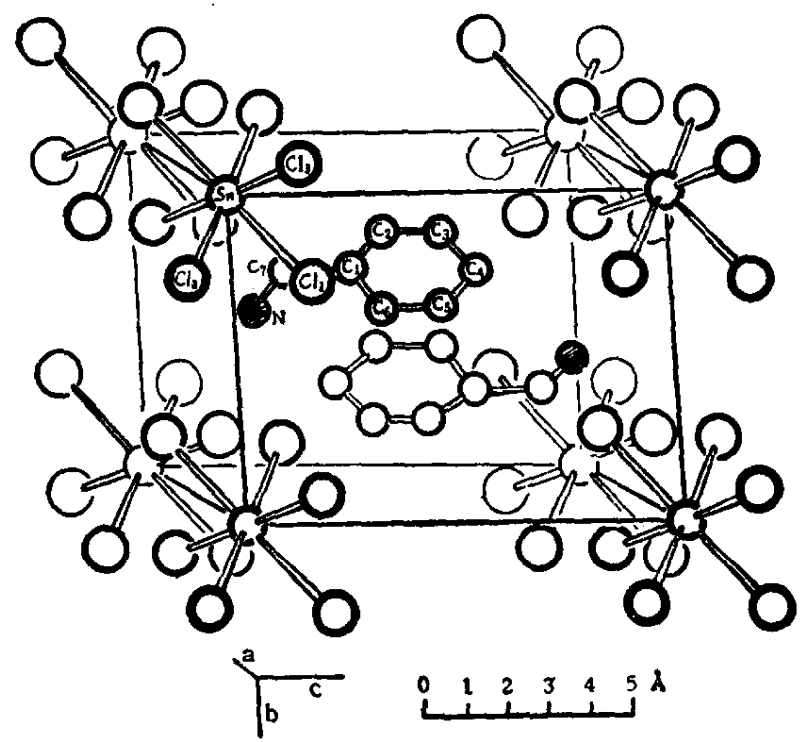

娄 3 晶体的結构

表 1 原子的参数

\begin{tabular}{llll}
\hline & \multicolumn{1}{c}{$x$} & \multicolumn{1}{c}{$y$} & \multicolumn{1}{c}{$z$} \\
\hline $\mathrm{Sn}$ & 0 & 0 & 0 \\
$\mathrm{Cl}_{1}$ & 0.817 & 0.270 & 0.155 \\
$\mathrm{Cl}_{2}$ & 0.303 & 0.810 & 0.140 \\
$\mathrm{Cl}_{3}$ & 0.800 & 0.850 & 0.117 \\
$\mathrm{C}_{1}$ & 0.292 & 0.278 & 0.324 \\
$\mathrm{C}_{2}$ & 0.242 & 0.158 & 0.406 \\
$\mathrm{C}_{3}$ & 0.250 & 0.158 & 0.539 \\
$\mathrm{C}_{4}$ & 0.275 & 0.297 & 0.596 \\
$\mathrm{C}_{8}$ & 0.323 & 0.417 & 0.518 \\
$\mathrm{C}_{8}$ & 0.318 & 0.417 & 0.384 \\
$\mathrm{C}_{7}$ & 0.300 & 0.274 & 0.180 \\
$\mathrm{~N}$ & 0.350 & 0.407 & 0.133 \\
\hline
\end{tabular}


上述結果确定中間物系由离子 $\left[\mathrm{SnCl}_{6}\right]^{2-}$ 和 $\left[\mathrm{C}_{6} \mathrm{H}_{5} \mathrm{CH}=\mathrm{NH}_{2}\right]+$ 所組成, 其化学式为 $\left[\mathrm{C}_{6} \mathrm{H}_{5}-\mathrm{CH}\right.$ $\left.=\mathrm{NH}_{2}\right]_{2} \mathrm{SnCl}_{6}$, 这一結果与 Meerwein 的推測相符 合。离子的鍵长和鍵角的近似值示出于圖 4 中。摛子 $\left[\mathrm{SnCl}_{6}\right]^{2-}$ 为一具有八面体构型的絡合离子; $\mathrm{Sn}$ $\mathrm{Cl}=2.44-2.52 \AA$, 而有机离子中全部原子形成一个 共面的共轭体系, 其中与苯环相連的 $\mathrm{C}-\mathrm{C}$ 单 鍵 估 計将趋于縮短 $(1.50 \AA$ )。这两个离子通过 $\mathrm{N}-\mathrm{H} \cdots \cdots$. $\mathrm{Cl}_{1}=3.07 \AA, \mathrm{N}-\mathrm{H} \cdots \cdots \mathrm{Cl}_{2}=3.01 \AA$ 的昰鍵結合在一 起, 其作用力除离子一离子鍵力外份有离子一偶極子力。
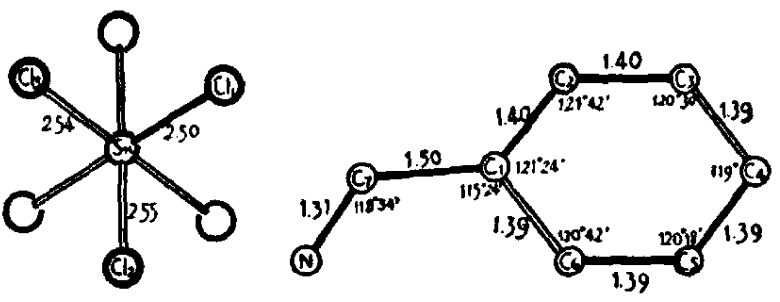

漓子 $\left.4 \mathrm{SnCl}_{6}\right]^{2-}$ 和 $\left[\mathrm{C}_{6} \mathrm{H}_{5}-\mathrm{CH}=\mathrm{NH}_{2}\right]+$ 的松型

中䦭物的晶体結构指出 Stephen 反应的反应方 程烒为

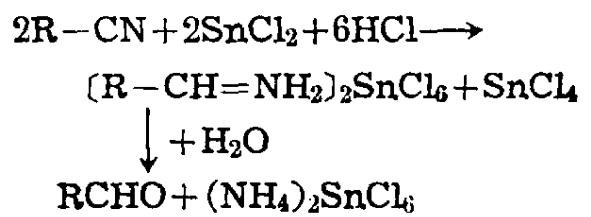

这一反应的产往往往胜物的类型而变化。在脂肪

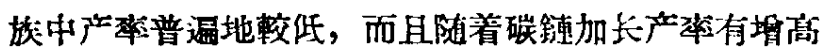
的趋问 [4]。这除与其他因素有关外，亦可能与䤈亚胺 离子本身的結构和稳定性以及中閒物中正負离子的相 互作用、空閵堆积, 亦即能否形成稳定的中閵物唱体 住关。

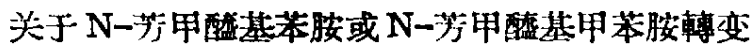
为酲的 Sonn 和 Müller 方法[3]的中間物的結构和反 应机构，也可参考本工作的結果。

周公度 陆照發 唐有稘 (北京大学化学采, 中国科学院应用物理研究所) 1957 年 6 月 14 日

[1] J. Stepher, J. Chem. Soc., 127, 18:4 (1925).

[2] Mecrwein, 見 Bayer, "Methoden der organischen Chemie", Band II, Teil 1, s. 299 (1954).

[3] E. Mosettig, "Organic Reactions" Vol. VIII(1954). [4] L. Turner, J. Chem. Soc., 1686 (1956).

\section{多分散性对聚甲基丙烯酸甲酯 在丙团中第二維利系数的影响}

融分子滨液渗透压的潧度依賴关系式

$$
\pi=C R T\left(\frac{1}{\bar{M}_{n}}+A_{2} C+\cdots \cdots\right)
$$

中第二維利系数 $A_{2}$ 的数值表征溶波中省分子綫的周

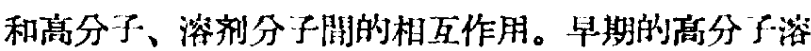
波理論認为 $A_{2}$ 半不依从于分子量，但买驗事头指出

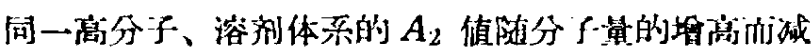

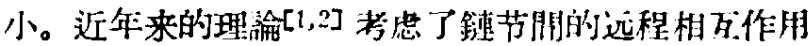

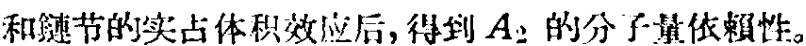
抹且說明从渗透还数据得到的 $A_{2}$ 和从光散射数据得 到的 $A_{2}^{\prime}$ ，只有单分散的試佯题等闰的。因此从实駿数 括来观祭 $A_{2}$ 对分子量的伐賴关系，必少进一少了解 多分散性对 $A_{2}$ 和 $A_{2}^{\prime}$ 值的影响。

Krigbaum 和 Flory[3] 留用聚异厂烯的两个分 粐試样及其混合物在环已烷中进行渗透压测定，得到

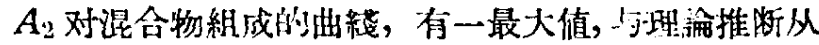
質地相符合。現在作等用两个粩过三次分級的聚 甲基间烯酸甲醌級份（級份 $1: \bar{M}_{n}=8.6 \cdot 10^{4}, \bar{M}_{w}=$ 9.2・104; 級份 $\left.2: \vec{M}_{n}=1.05 \cdot 10^{6}, \bar{M}_{w}=1.18 \cdot 10^{6}\right)$ 及 其混合物在两国中 $25^{\circ} \mathrm{C}$ 时进行渗透山太光敬射測分， 得到的結果如圖 1 。圖中 $A_{2}$ 值对混合物絹成（朋級份

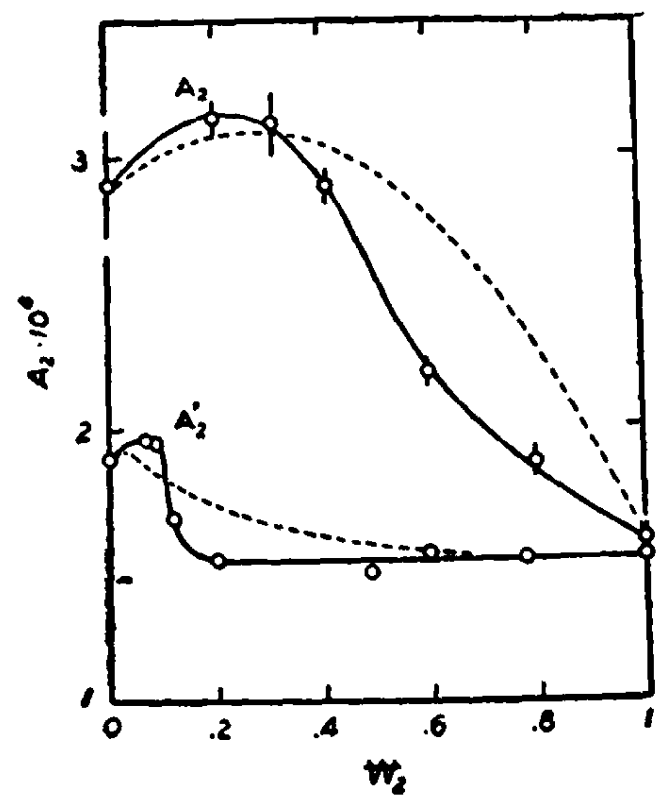

1 湜合級份的 $A_{2}, A_{2}^{\prime}$ 对面量分数的圆

2 的重量百分数 $w_{2}$ 表示)的曲榑在 $w_{2}=0.2$ 处有一筧 的高峰, 而 $A_{2}^{\prime}-w_{2}$ 馢則在 $w_{2}=0.06$ 处有一较狹的高 峰。純級份 2 的 $A_{2}$ 和 $A_{2}^{\prime}$ 值㯊为接近, 但是純級份 1 的 $A_{2}$ 值要比 $A_{2}$ 值大 1.5 倍, 其原因目前作者等还不

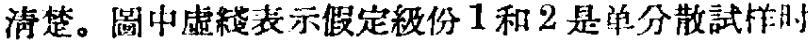
混合物的 $A_{2}$ (和 $A_{2}^{\prime}$ ) 对組成的曲綫。按照 Flory [1,4] 或 Grimley[2]的理諭，一个单分散藏样的

$$
A_{2}=K F(X) \text {, }
$$

式中 $K$ 可分于量無关， $A_{2}$ 的分子量体赖性由复杂函 数 $F(X)$ 决定。对两个单分散試併的混合物，

$$
A_{2}=K \sum_{i} \sum_{j} w_{i} w_{j} F\left(X_{i j}\right),
$$

